\title{
Observing metamaterial induced transparency in individual Fano resonators with broken symmetry
}

\author{
Ranjan Singh, ${ }^{1,2, a)}$ Ibraheem A. I. Al-Naib, ${ }^{3}$ Yuping Yang, ${ }^{2}$ Dibakar Roy Chowdhury, ${ }^{1}$ \\ Wei Cao, ${ }^{2}$ Carsten Rockstuhl, ${ }^{4}$ Tsuneyuki Ozaki, ${ }^{3}$ Roberto Morandotti, ${ }^{3}$ and Weili Zhang ${ }^{2, b)}$ \\ ${ }^{1}$ Center for Integrated Nanotechnologies, Materials Physics and Applications Division, Los Alamos National \\ Laboratory, Los Alamos, New Mexico 87545, USA \\ ${ }^{2}$ School of Electrical and Computer Engineering, Oklahoma State University, Stillwater, Oklahoma 74078, \\ USA \\ ${ }^{3}$ INRS-EMT, Université du Québec, Varennes, Québec J3X 1S2, Canada \\ ${ }^{4}$ Institute of Condensed Matter Theory and Solid State Optics, Friedrich-Schiller-Universität Jena, \\ Jena 07743, Germany
}

(Received 22 September 2011; accepted 20 October 2011; published online 15 November 2011)

\begin{abstract}
Metamaterial induced transparency is demonstrated using individual split ring resonators with two gaps on opposite side. For the symmetric structure, only a low quality dipolar resonance is witnessed at a normal incidence excited with electric field along the resonator gaps. Displacement of one gap from the centre breaks the symmetry and a higher order mode, inaccessible in the symmetric structure, is excited. Coherent interaction among the modes in the split ring resonator forms an extremely sharp narrowband transparency window centred directly at the dipole resonance. Such metamaterial could facilitate coherent manipulation of terahertz signals for delay, storage, and nonlinear applications. (C) 2011 American Institute of Physics. [doi:10.1063/1.3659494]
\end{abstract}

Metamaterials attracted a lot of research interest in recent years due to their versatile characteristics and the potential to implement a plethora of applications such as perfect lenses, sensors, antennas, frequency selective surfaces, and modulators. ${ }^{1-9}$ Most importantly, the resonators that constitute the metamaterial can be designed at will such that they support resonances at any electromagnetic frequency and with any multipolar radiation pattern. ${ }^{1}$ Intriguingly, the scattering properties of the resonators could be forced to correspond not just to an electric dipole, but also to an electric quadrupole, magnetic dipole, or any other higher order mode. This fundamentally altered the perception to think about the design of complex resonators since they could be also designed to support resonances with largely disparate properties. In the context of the present work, the property that matters most is the life-time. Resonators supporting a broad resonance are usually called bright since they couple well to free space. Their nearfield is characterized in most cases by electric dipolar patterns. Resonators supporting a narrow resonance are usually called dark since they do couple only weakly to free space. Their near-field is usually characterized by an electric quadrupolar pattern. In the extreme case, such resonators cannot radiate at all, i.e., if forbidden by symmetry constraints. They would live entirely in the near-field and would only be damped by non-radiative dissipation. The latter can be made very small if the resonators are operated at low frequencies where most metals can be considered as close to perfect conductors. Combining different resonators to form new unit cells gave impetus to the entire field of metamaterials and triggered many genuine ideas.

Most notably, it led to the implementation of structures that bear analogy to electromagnetically induced transparency (EIT). This effect is usually observed in carefully selected

\footnotetext{
${ }^{\text {a)} E l e c t r o n i c ~ m a i l: ~ r a n j a n @ l a n l . g o v . ~}$

b)Electronic mail: weili.zhang@okstate.edu.
}

three level atomic systems excited with dressed states. Such classical field interference of quantum type has been proposed and demonstrated by several groups as a means to cancel absorption of electromagnetic wave propagating through artificially designed metamaterials at a desired frequency. ${ }^{10-30}$ Thus, the EIT effect caused by wave propagation in metamaterials was termed as metamaterial induced transparency. ${ }^{19}$ To observe metamaterial induced transparency, two resonators are usually combined into a single unit cell. They absorb light at the same frequency; hence, the medium is opaque. One resonator usually acts as a highly radiative "bright" resonator and is directly excited by the incident electromagnetic wave. The second resonator is non-radiative "dark" and is indirectly excited through coupling with the bright resonator. The coherent interaction among both resonators leads to the desired well-defined narrow transparency window due to a coherent cancellation of the extinction.

In this letter, we show that the metamaterial induced transparency can be evoked in a single terahertz Fano resonator consisting of a square two-gap split ring resonator (SRR) array excited at normal incidence by an electric field which is parallel to the gap. In none of the previous schemes, a transparency peak could be excited in a single two-gap resonator by maintaining the electric field along the gaps, albeit a Fano type resonance was earlier observed by exciting an asymmetric two-gap resonator with an electric field perpendicular to the gaps. ${ }^{11-13,22}$ For the structure under investigation, a broad resonant feature is supported when the SRRs are perfectly symmetric with the two gaps being placed exactly along the central vertical axis. As soon as one of the gaps is displaced from its central position, a high quality $(Q)$ factor transparency window opens up at exactly the same frequency where the broad resonance occurred. In this scheme, for induced transparency, the two wire elements adjacent to the SRR gaps are directly excited by the incident wave. When the gaps are in a central position, the wire arms of the resonator resonate at 

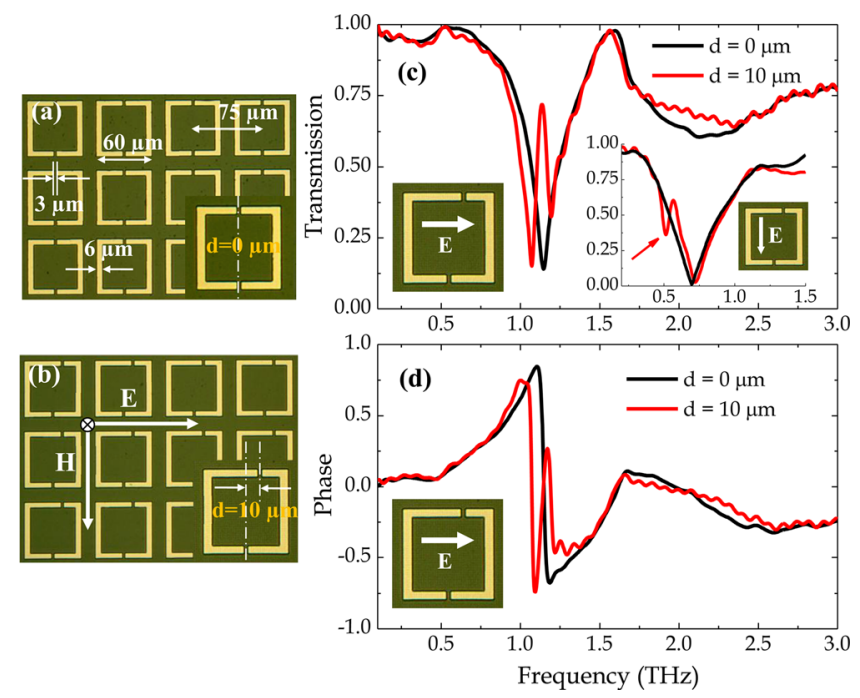

FIG. 1. (Color online) Microscopic image of the (a) Symmetric metamaterial, SR with $\mathrm{d}=0 \mu \mathrm{m}$ and (b) Asymmetric metamaterial, ASR2 with $\mathrm{d}=10 \mu \mathrm{m}$. Measured (c) transmission and (d) phase spectra of SR and ASR2 when the electric field is parallel to the gap. The inset of (c) shows the spectra of SR and ASR2 when the electric field is perpendicular to the gap arms.

an identical frequency and contribute to the formation of a broad background caused by the dipolar nature of the resonances in each wire. The currents in these two arms oscillate inphase for most part of the spectrum and interfere constructively. But as soon as one of the gaps is off-centered, their resonance frequencies differ slightly, leading to a strong coupling between the arms of the SRR. Intriguingly, they oscillate out-of-phase for a narrow range of frequencies leading to a destructive interference. Such configuration causes a sharp transparency sub-band in the background spectrum of the broad dipole resonance. The most remarkable feature of metamaterial induced transparency is the drastic reduction in the group velocity of the electromagnetic wave passing through such a material, mainly achieved within a high $Q$-factor, lossless transparency window. This property can facilitate the slowing down or the storage of light making it extremely important for the design of the buffers and delay lines required for future terahertz communication systems as well as for nonlinear signal processing.

In the experiments described here, we fabricated four sets of planar two-gap SRR samples starting from a perfect symmetric one with both the top and the bottom gaps exactly at the center (see Fig. 1(a)) and then gradually displaced the upper gap by a distance " $d$ " while keeping the lower gap fixed, in order to break the symmetry (Fig. 1(b)). In the four different metamaterial samples, the top gap was shifted by $d=0,5,10$, and $20 \mu \mathrm{m}$, thus we address them as SR, ASR 1 , ASR2, and ASR3, respectively. All four of the two-gap SRR metal film patterns were fabricated using photolithographic techniques, followed by the depositions of $200 \mathrm{~nm}$ of aluminum on top of a $640 \mu \mathrm{m}$ thick n-type silicon substrate $(\varepsilon=11.68)$. The detailed geometrical dimensions of the SRRs are shown in Fig. 1(a). The measurement was carried out using a typical $8 f$ confocal terahertz time-domain spectroscopy (THz-TDS) system..$^{31,32}$

Figure 1(c) shows the measured transmission spectra of a perfect symmetric metamaterial (SR) with $d=0 \mu \mathrm{m}$ and an asymmetric SRR (ASR2) with $d=10 \mu \mathrm{m}$. A sharp transparency window opens up in the spectrum of ASR2. The corresponding measured phase spectra are shown in Fig. 1(d). The complementary measurements for phase and transmission are consistent with the natural properties of a causal system. The metamaterial array used here is a linear system in which the real and imaginary parts of the transfer function are related through the Kramers-Kronig relations. The sudden phase change leads to a strong dispersion in the medium. This sharp phase change is the key to achieve a slow-light behavior due to reduced group velocities. The inset of Fig. 1(c) is the measured transmission for SR and ASR2 with the electric field polarized perpendicular to the gap bearing arms. We clearly observe one single broad resonance at $0.7 \mathrm{THz}$ for the SR metamaterial but two resonances for the ASR2. A sharp Fano resonance at $0.5 \mathrm{THz}$ is excited due to symmetry breaking in ASR2. This sharp resonant feature at terahertz frequencies has been discussed in detail in Ref. 13 and is not of further relevance to the present work.

In Fig. 2, we show the measurements and the numerical simulations for all the four samples. ${ }^{33}$ Starting from SR $(d=0 \mu \mathrm{m})$, where only a single resonance dip is observed in Fig. 2(a) at $1.13 \mathrm{THz}$, we move to an asymmetric SRR sample, specifically the ASR 1 with $d=5 \mu \mathrm{m}$ in which the transparency peak opens up as shown in Fig. 2(b) at exactly the same frequency $(1.13 \mathrm{THz})$ at which the dipole resonance dip occurred in the perfectly symmetric sample. It is important to notice the drastic difference in terms of quality factor of the broad dipole resonance $(Q=2.8)$ and the narrow transparency sub-band resonance window which has an extremely high $Q$ factor of 68 . When the top gap is further shifted by $d=10 \mu \mathrm{m}$, the transparency resonance band broadens as well as the transmission increases in Fig. 2(c), and for
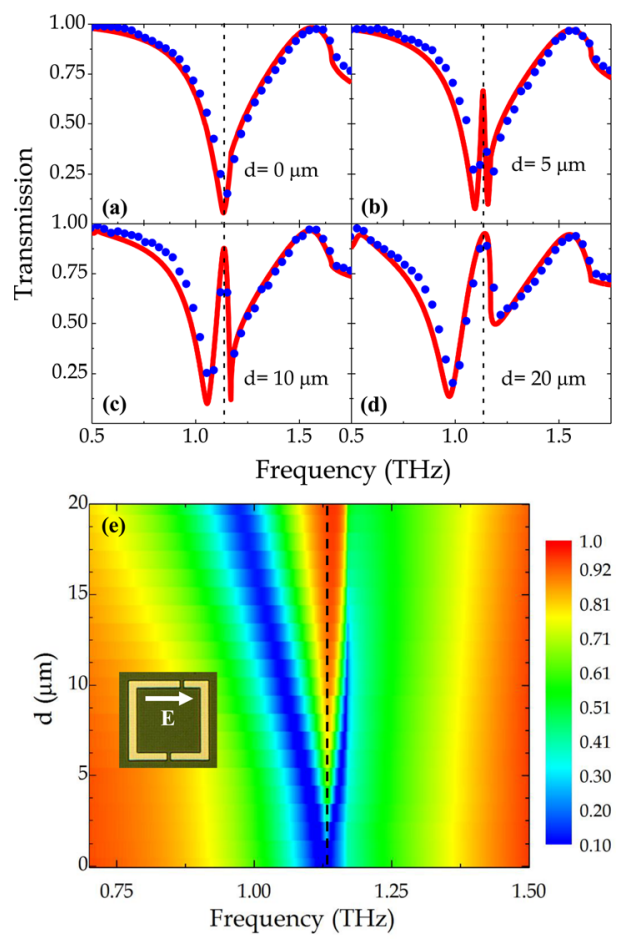

FIG. 2. (Color online) Measured (blue solid circle) and simulated (red curve) transmission amplitude spectra for (a) SR, (b) ASR1, (c) ASR2, and (d) ASR3 (e) The simulated transmission amplitude of SRR as a function of asymmetry parameter ' $d$ '. 


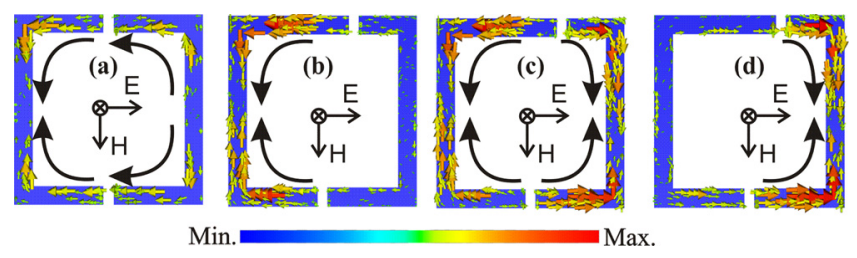

FIG. 3. (Color online) Simulated current distributions at resonance frequencies of (a) SR at $1.13 \mathrm{THz}$, (b) ASR2 at $1.05 \mathrm{THz}$, (c) ASR2 at $1.13 \mathrm{THz}$, and (d) ASR2 at $1.17 \mathrm{THz}$.

$d=20 \mu \mathrm{m}$, the transparency peak finally reaches $96 \%$ in Fig. 2(d). The simulated transmission is in good agreement with most of the measured transmission spectra, though for the low asymmetry structure in Fig. 2(b), the measured EIT resonance band could not exactly match the simulation due to the limited resolution of our measurement system.

Figure 2(e) shows the simulated transmission where the splitting of the single dipole resonance is observed as soon as the symmetry of the SRR structure is broken even when the SRR top gap is shifted by a minute distance of $d=1 \mu \mathrm{m}$. The $y$-axis shows the top gap shift of the SRR as function of the parameter " $d$." As the distance " $d$ " is increased the resonance mode splitting leading to a transparency in the spectrum at around $1.13 \mathrm{THz}$ becomes more evident since the transparency window broadens and its amplitude nearly reaches 1 . The asymmetry parameter " $d$ " leads to the broadening and enhancement in transparency of the EIT window centered at a single frequency as shown by the dotted line in Fig. 2. Thus, " $d$ " acts as the control parameter in this metamaterial system.

To gain further understanding on the nature of these resonances, we looked at the surface current distributions at the resonances of the symmetric, SR, and the asymmetric metamaterial, ASR2, in Fig. 3. At the symmetric dipole resonance frequency of the SR as shown in Figs. 3(a) the two symmetric current loops at the top and the bottom arms are parallel to each other with nodes at the center of both vertical arms. The terahertz field scattered from the SR interferes constructively and gives rise to a strong scattering leading to a broad dipole resonance feature. The parallel surface currents are highly radiative in nature.

Once the symmetry is broken, for example, in metamaterial ASR2, the dipole mode of the SR splits in two distinct resonances at $1.05 \mathrm{THz}$ and $1.17 \mathrm{THz}$. The simulations in Figs. 3(b)-3(d) show the surface currents at the lower frequency resonance of $1.05 \mathrm{THz}$, at the transparency peak of $1.13 \mathrm{THz}$ and the higher frequency resonance at $1.17 \mathrm{THz}$. In Fig. 3(b), the currents at the lower resonance frequency are excited only in the left hand side longer wire arm of the ASR2. In this left arm, there are two parallel currents which form a dipole and give rise to the resonant effect at $1.05 \mathrm{THz}$. At the higher frequency resonance $(1.17 \mathrm{THz})$, Fig. 3(d) reveal that the right side smaller wire arm is excited with parallel currents characterized by a stronger field confinement, thus leading to a sharper or a higher $Q$ resonance compared to the longer left side wire.

In Fig. 3(c), the current distribution associated with the transparency resonant peak at $1.13 \mathrm{THz}$, we observe antiparallel current pairs which are also anti-symmetric in nature causing a destructive interference of the scattered fields. This destructive interference between the radiated fields arising from the anti-symmetric currents completely suppresses the scattering allowing the incident wave to be transmitted without losses - thus resulting in an EIT effect.

In conclusion, we have experimentally and numerically demonstrated metamaterial induced transparency in a single asymmetric terahertz Fano resonator array. At a low degree of asymmetry, the transparency window is extremely sharp and is accompanied by steep phase change which could be exploited to design slow-light terahertz devices and to induce an enhancement of the terahertz nonlinearities. Higher degree of asymmetry allows for the tuning of the amplitude and bandwidth of the transparency window.

${ }^{1}$ J. B. Pendry, A. Holden, D. Robbins, and W. Stewart, IEEE Trans. Microwave Theory Tech 7, 2075 (1999).

${ }^{2}$ R. A. Shelby, D. R. Smith, and S. Schultz, Science 292, 5514 (2001).

${ }^{3}$ T. J. Yen, W. J. Padilla, N. Fang, D. C. Vier, D. R. Smith, J. B. Pendry, D. N. Basov, and X. Zhang, Science 303, 1494 (2004).

${ }^{4}$ C. M. Soukoulis, S. Linden, and M. Wegener, Science 315, 47 (2007).

${ }^{5}$ J. F. O'Hara, R. Singh, I. Brener, E. Smirnova, J. Han, A. J. Taylor, and W. Zhang, Opt. Express 16, 1786 (2008).

${ }^{6}$ A. I. Al-Naib, C. Jansen, and M. Koch, Appl. Phys. Lett. 93, 083507 (2008).

${ }^{7}$ B. Lahiri, A. Z. Khokhar, R. M. De La Rue, S. G. McMeekin, and N. P. Johnson, Opt. Express 17, 1107 (2009)

${ }^{8}$ R. Singh, C. Rockstuhl, C. Menzel, T. P. Meyrath, M. He, H. Giessen, F. Lederer, and W. Zhang, Opt. Express 17, 9971 (2009)

${ }^{9}$ H. T. Chen, W. J. Padilla, J. M. O. Zide, A. C. Gossard, A. J. Taylor, and R. D. Averitt, Nature 444, 597 (2006).

${ }^{10}$ S. Zhang, D. A. Genov, Y. Wang, M. Liu, and X. Zhang, Phys. Rev. Lett. 101, 047401 (2008).

${ }^{11}$ S. Prosvirnin and S. Zouhdi, "Resonances of closed modes in thin arrays of complex particles," in Advances Electromagnetics of Complex Media and Metamaterials, S. Zouhdi et al., ed. (Kluwer Academic Publishers, USA, 2003), pp. 281-290.

${ }^{12}$ V. A. Fedotov, M. Rose, S. L. Prosvirnin, N. Papasimakis, and N. I. Zheludev, Phys. Rev. Lett. 99, 147401 (2007).

${ }^{13}$ R. Singh, I. A. I. Al-Naib, M. Koch, and W. Zhang, Opt. Express 19, 6312 (2011).

${ }^{14}$ N. Papasimakis, V. A. Fedotov, N. I. Zheludev, and S. L. Prosvirnin, Phys. Rev. Lett. 101, 253903 (2008).

${ }^{15}$ R. Singh, C. Rockstuhl, F. Lederer, and W. Zhang, Phys. Rev. B 79, 085111 (2009).

${ }^{16}$ N. Liu, L. Langguth, T. Weiss, J. Kastel, M. Fleischhauer, T. Pfau, and H. Giessen, Nature Mater. 8, 758 (2009).

${ }^{17}$ S. Y. Chiam, R. Singh, C. Rockstuhl, F. Lederer, W. Zhang, and A. A. Bettiol, Phys. Rev. B 80, 153103 (2009).

${ }^{18}$ P. Tassin, L. Zhang, T. Koschny, E. N. Economou, and C. M. Soukoulis, Phys. Rev. Lett. 102, 053901 (2009).

${ }^{19}$ N. Papasimakis and N. I. Zheludev, Opt. Photonics News 20, 22 (2009).

${ }^{20}$ V. Yannopapas, E. Paspalakis, and N. V. Vitanov, Phys. Rev. B 80, 035104 (2009).

${ }^{21}$ B. Luk'yanchuk, N. I. Zheludev, S. A. Maier, N. J. Halas, P. Nordlander, H. Giessen, and C. T. Chong, Nat. Mater. 9, 707 (2010)

${ }^{22}$ R. Singh, I. A. I. Al-Naib, M. Koch, and W. Zhang, Opt. Express 18, 13044 (2010)

${ }^{23}$ Z.-G. Dong, H. Liu, J.-X. Cao, T. Li, S.-M. Wang, S.-N. Zhu, and X. Zhang, Appl. Phys. Lett. 97, 114101 (2010)

${ }^{24}$ J. Wu, B. Jin, J. Wan, L. Liang, Y. Zhang, T. Jia, C. Cao, L. Kang, W. Xu, J. Chen, and P. Wu, Appl. Phys. Lett. 99, 161113 (2011)

${ }^{25}$ K. Aydin, I. M. Pryce, and H. A. Atwater, Opt. Express 18, 13407 (2010)

${ }^{26}$ A. E. Çetin, A. Artar, M. Turkmen, A. A. Yanik, and H. Altug, Opt. Express 19, 22607 (2011)

${ }^{27}$ R. Singh, X. Lu, J. Gu, Z. Tian, and W. Zhang, J. Opt. 12, 015101 (2010)

${ }^{28}$ H. Xu, Y. Lu, Y. P. Lee, and B. S. Ham, Opt. Express 18, 17736 (2010)

${ }^{29}$ Y. Ma, Z. Li, Y. Yang, R. Huang, R. Singh, S. Zhang, J. Gu, Z. Tian, J. Han, and W. Zhang, Opt. Mat. Express 1, 391 (2011)

${ }^{30}$ Z. Li, Y. Ma, R. Huang, R. Singh, J. Gu, Z. Tian, J. Han, and W. Zhang, Opt. Express 19, 8912 (2011)

${ }^{31}$ D. Grischkowsky, S. Keiding, M. van Exter, and C. Fattinger, J. Opt. Soc. Am. B 7, 2006 (1990).

${ }^{32}$ M. He, A. K. Azad, S. Ye, and W. Zhang, Opt. Commun. 259, 389 (2006).

${ }^{33}$ CST Microwave Studio ${ }^{\circledR}$, (http://www.cst.com). 